\title{
Unainiciación deportiva sin lesiones, estudio con entrenadores de fútbol y fútbol sala Asports initiation without injury, studio football coaches and football
}

\author{
Armando Costa*, Oleguer Camerino**, Pedro Sequeira*** \\ *Instituto Superior de Ciências Educativas (Portugal), **INEFC-Universidad de Lleida (España), *** Escola Superior de Desporto de Rio \\ Maior (ESDRM) (Portugal)
}

Resumen. La iniciación deportiva ha de estimular no sólo estilos de vida activos y saludables sino también la prevención de lesiones y riesgos innecesarios para los jóvenes practicantes. En este artículo, aplicando la metodología observacional, registramos las intervenciones de prevención de lesiones de una muestra representativa de entrenadores $(n=8), 4$ de fútbol y 4 de fútbol sala de clubs grandes y pequeños. Diseñamos un sistema de observación ad hoc (SOCTAS), para el registro sistemático de la actuación de estos entrenadores en sus contextos naturales. El resultado nos muestra que los entrenadores de fútbol y fútbol sala de clubs grandes, con relación a los clubs pequeños, son los que promueven más conductas saludables y de evitación de lesiones mediante la transmisión de: feedback correctivos, crítica positiva e informaciones a los jugadores sobre prevenciones. Palabras claves. fútbol base, lesiones deportivas, entrenamiento, competición.

Abstract. Organised sports for children in the initiation stage must bring health benefits, and coaches should promote the health of their young participants through interventions designed to avoid unnecessary risks. In this study, we used observational methodology to detect actions that reflect the importance that soccer and futsal coaches from small and large clubs attach to health-related aspects. We observed 8 coaches ( $\mathrm{n}=8$ ), equally distributed among these sports and the type of club analyzed. We designed an ad hoc category system (SOTCAS) for the systematic recording of the actions of these coaches in their natural settings. Our results show that soccer and futsal coaches from the larger clubs are more active in promoting healthy practices and preventing injury through the provision of corrective feedback, constructive criticism, and advice about prevention.

Key words. sports for children, sports injuries, training, competition

\section{Introducción}

\section{Condiciones de la iniciación deportiva}

La juventud es un periodo importante para el desarrollo de hábitos y actitudes saludables ya que los jóvenes consolidan los estilos de vida activos en esta etapa (Biddle, Sallis \& Cavill, 1998; Cavill, 2001). Las actividades físicas extraescolares por lo menos 3 veces por semana con intensidad baja pueden prevenirlos de enfermedades vasculares y luchar contra una obesidad creciente; muchas investigaciones han reconocido la necesidad de incorporar la actividad física en estas edades para asegurar el bienestar corporal. (Ara, et al. 2006; Ortega, Ruiz, Castillo \& Sjöström, 2008).

No obstante, se constata que el nivel de práctica de actividad física en estas edades se encuentra en retroceso (Gorely, Marshall, Biddle \& Cameron, 2007). Diferentes estudios verifican que los jóvenes sólo mantienen niveles de actividad física deseables los días que practican un deporte dirigido (Wickel \& Eisenmann, 2007), destacando así la importancia de la práctica deportivas organizadas con unas condiciones determinadas (Janssen, et al. 2006; Rivas, et al. 2012).

La iniciación deportiva debe reunir unas condiciones; objetivos alcanzables y realista para sus practicantes que les permita demostrar su competencia personal (Peiró, Devís, Beltrán \& Fox, 2008); existencia de una organización deportiva que proporcione satisfacciones sociables (Devís, Beltrán \& Fox,2013); una intensidad asumible moderada a vigorosa administrada entre 3 a 5 veces por semana (Fairclough, Boddy, Mackintosh, Valencia-Peris \& Ramirez-Rico, 2014); vivencia de relaciones interpersonales y de participación en la actividad (Haskell, et al. 2007).

\section{Una iniciación deportiva del fútbol sin riesgos}

Cuando analizamos el entrenamiento dirigido a jóvenes nos encontramos con un enfoque de rendimiento; la mayoría de los estudios en este ámbito se centran en la eficacia del deportista para obtener elevados niveles de rendimiento. Los planteamientos de una iniciación deportiva que atienda una prevención de lesiones no está suficientemente extendi-

Fecha recepción: 28-04-14- Fecha envío revisores: 29-04-14- Fecha de aceptación: 04-07-14 Oleguer Camerino Foguet ocamerino@inefc.es do en la literatura científica, en la actualidad encontramos en los terrenos de juego carencias en la orientación de los programas y pocas previsiones con relación a la evitación de lesiones y de riesgo por parte de los entrenadores que siguen teniendo a sus jugadores subyugados bajo su excesiva autoridad (Chelladurai, 1990; Cruz \& Gomes, 1996).

Las lesiones en el fútbol se siguen produciendo e incluso agravando, aunque está comprobado que estas lesiones son menores en el fútbol base y que se incrementan con la edad (Kibler, 1995). Esta tendencia hace que los profesionales de la salud que trabajan en el ámbito deportivo manifiesten preocupación(Olmedilla,Andreu, Ortín \& Blas, 2009).

El entrenador del fútbol base tiene que planificar y organizar sus entrenamientos promoviendo actividades que eviten estas lesiones e incorporen conductas y que conciencen a sus jugadores de la importancia de la prevención de lesiones, teniendo especiaal cuidado en los proceso de preparación y calentamiento de la competición (Bompa, 1999). El entrenamiento debe ser programado de acuerdo a las competencias del grupo y la etapa de maduración fisiológica de los participantes para poder maximizar sus aprendizajes técnicos que incluyan hábitos de vida saludables y prevenciones de lesiones futuras (Añó, 1997).

Deste este modo, el entrenador de jóvenes tiene que desarrollar un estilo de trabajo en esta edades que no imite a los equipos de alta competición (Buceta, 2001). Para ello es importante que los entrenadores trabajen con formas jugadas y situaciones de decisión que incluyan el control de las cargas de trabajo y la inclusión de estiramientos y ejercicios de equilibrio propioceptivo (Gioftsidou \& Malliou, 2006)

El objetivo del presente trabajo se centra en registrar y analizar los comportamientos de entrenadores de fútbol base que tienen como finalidad preveer las lesiones de los jugadores en sus entrenamientos. Se pretende contrastar también si existen diferencias en estos comportamientos en relación a la envergadura de la organización deportiva dónde trabajan y al mismo tiempo en relación a las modalidades de fútbol y fútbol-sala. Así pretendemos obtener patrones de conducta de una muestra representativa de los técnicos de fútbol base para describir su perfil conductual hacia la prevención de lesiones y riesgos de sus jugadores.

Método

Utilizamos la Metodología Observacional (Anguera, 1990) que se adapta a las características necesarias de nuestro estudio desarrollado en un contexto natural (Anguera, 2003), lo que excluye cualquier tipo de 
intervención y de manipulación. Por ello elaboramos ad hoc (Anguera, Blanco \& Losado, 2001) un sistema de categorías (SOCTAS) que nos permitió observar las conductas de los entrenadores de jóvenes deportistas en el transcurdo del proceso de entrenamiento. El diseño observacional es diacrónico del tipo (S/N/M); de seguimiento (observaciones seguidas de varios entrenamientos), nomotético (observación de entrenadores de fútbol y fútbol sala considerados como dos unidades de observación independientes) y multidimensional (niveles de respuesta diferenciados registrados simultáneamente) (Anguera, Blanco, Mendo \& Losada, 2011)

\section{Participantes}

La muestra está compuesta por 8 entrenadores $(n=8)$ con una misma formación y experiencia técnica (tabla 1); cuatro de equipos de fútbol y cuatro de equipos de fútbol sala, y en cada una de estas tipologías fueron divididos en entrenadores de clubes de gran envergadura y entrenadores de clubes de pequeña envergadura. La diferenciación de estas organizaciones deportivas viene determinada por el volumen y número de fichas federativas según los criterios de otros estudios (Ford, Yates \& Williams, 2010). La edad de los jugadores está comprendida entre 6 a 12 años de las categorías alevín e infantil, que se repartieron equitativamente, $\mathrm{y}$ todos del género masculino de la región de Lisboa(Portugal).

\section{Procedimientos}

Para las filmaciones de los entrenamientos se utilizaron dos cámaras digitales (Samsung VP-D70 y Samsung VP-D301) para captar los comportamientos en su contexto natural. Para la realización de estas filmaciones se obtuvo el consentimiento informado de todos los participantes tanto de los jugadores como de los entrenadores. Se registraron cinco entrenamientos para cada uno de los entrenadores, dándonos un volumen total de 40 sesiones de entrenamiento lo que implicaba 35 horas de filmación. Las filmaciones se realizaron al principio de la temporada durante dos meses en los 50 primeros minutos de cada entrenamiento con una toma de cámara global y registrando la voz, el gesto del entrenador y la respuesta de sus jugadores.

\section{Instrumento de observación}

Para estudiar la multiplicidad de las conductas de estos entrenadores observados de manera sistemática, se construyó un sistema de categorías multidimensionales (SOCTAS) (ver tabla 1) compuesto por cinco macro criterios, nueve criterios y veintiuna categorías siguiendo los principios de exhaustividad y mutuamente exclusividad (E/ME). Para la definición de las categorías se utilizó la revisión de la literatura existente, conjuntamente con los datos obtenidos en la fase exploratoria para llegar a su validación (Anguera, 2003).

\section{Instrumento de registro}

Efectuamos un registro continuo de todas las sesiones grabadas con el programa informático LINCE (Gabín, Camerino, Castañer \&v Anguera, 2012) que es un software libre -descargado desde la web http://www.obseresport.com/-e interactivo multimedia, altamente flexible que permite visionar, registrar y categorizar en la misma pantalla del ordenador la grabación digitalizada de los vídeos de las sesiones observadas. Los observadores, que eran expertos y conocedores del fútbol, fueron entrenados hasta conseguir un consenso aceptable y superar una confiabilidad inter observador con un coeficiente kappa-obtenido mediante el programa SDIS-GSEQ versión 4.2 (Bakeman \& Quera, 1996, 2001)-de un valor de 0,99 que son aceptados en términos de estabilidad y consistencia (Castellano, Hernández, Gómez, Fontetxa \& Bueno, 2000)

\section{Análisis de los datos}

En primer lugar, se calculó la distribución de frecuencias y porcentajes de los distintos criterios del SOCTAS con el paquete estadístico IBM SPSS Statistics versión 20.0 (IBM Corp., Armonk, NY) y con un nivel de significación de $p<.05$.
Tabla 1

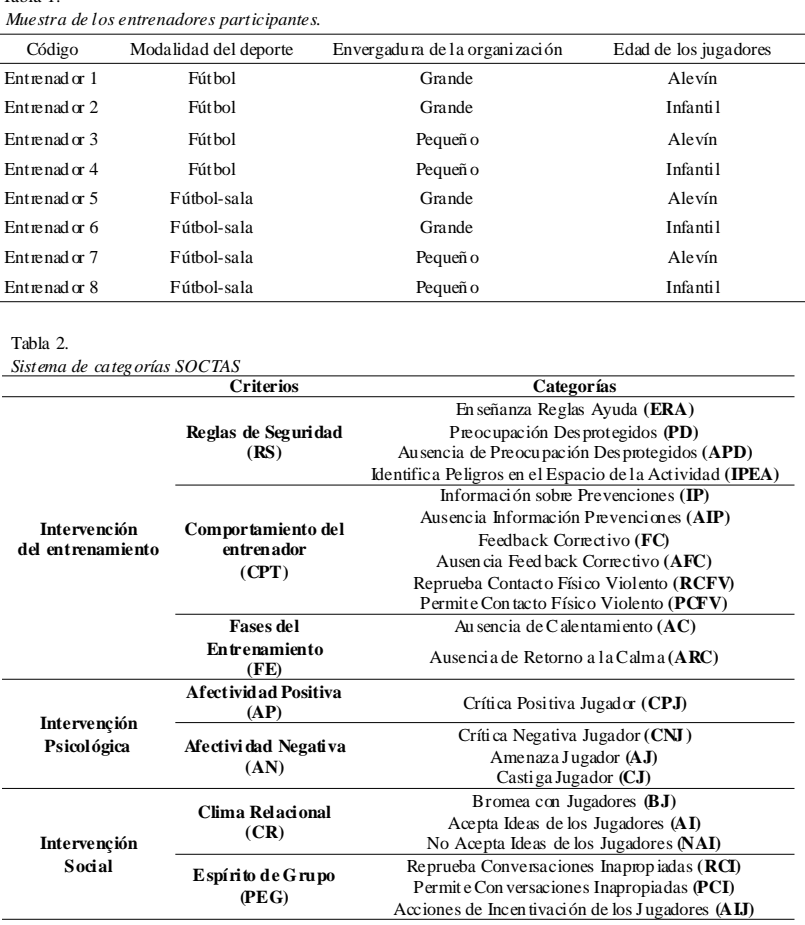

Además del estudio descriptivo, se procedió al análisis de T-patterns para obtener los patrones de conducta, tal como hemos establecido en los objetivos del estudio, utilizando el software THEME 6 (Magnusson, 1996, 2000, 2006). Este análisis permite obtener una relación concatenada temporal de conductas emergentes y no detectables con los métodos tradicionales de análisis de datos que se representan en gráficos en árbol (figura 2, 4, 6 y 8) o dendogramas (Camerino, Chaverri, Anguera \& Jonsson, 2012; Jonsson, et al. 2006, 2010).

\section{Resultados}

\section{Análisis estadístico descriptivo}

Primero presentamos los datos estadísticos descriptivos de cada uno de los entrenadores (Figura 1, 3, 5, 7 y 9), para después realizar un análisis de patrones temporales (T-patterns) con el programa THEME 6 (Magnusson, 1996, 2000), que nos proporcionará las asociaciones entre las conductas registradas según su coincidencia temporal. Los resultados de este análisis secuencial se expresan en un gráfico de árbol o dendogramas (figura 2, 4, 6 y 8) que ilustran las relaciones entre las conductas a lo largo de las sesiones de entrenamiento.

Comportamientos de entrenadores de fútbol de clubs pequeños

Los entrenadores de fútbol de organizaciones deportivas pequeñas tienden a establecer una relación ambivalente (fig. 1) con sus jugadores,

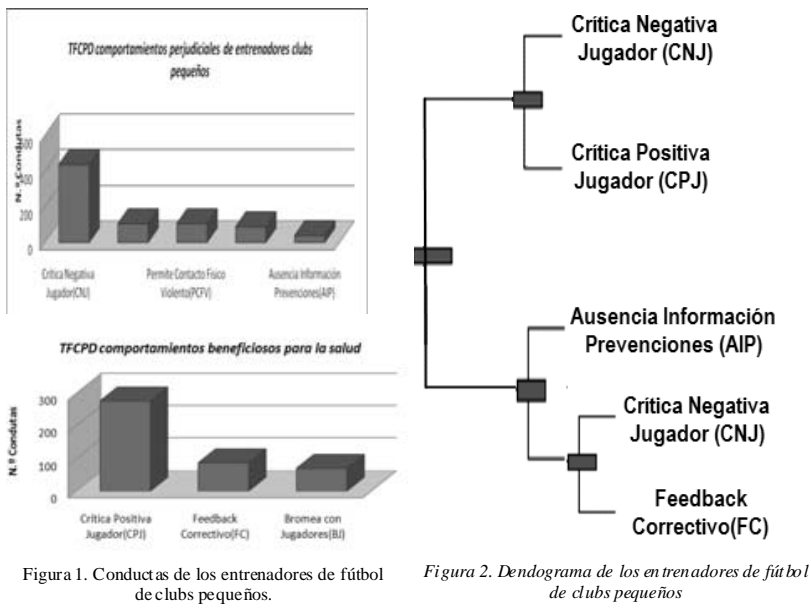


manifestando tanto crítica negativas ( $\mathrm{n}=438$ ), como actitudes positivas motivacionales ( $\mathrm{n}=227)$; hecho que condiciona de forma perjudicial el clima del entrenamiento. Esto queda corroborado por el acompañamiento de conductas permisivas en cuanto a conversaciones inapropiadas ( $\mathrm{n}=108)$, contactos violentos ( $\mathrm{n}=108$ ) y amenazas a los jugadores $(\mathrm{n}=91)$.

En el análisis del dendograma (fig. 2) podemos ver las secuencias conductuales más utilizadas por estos entrenadores que se basan en la combinación entre la crítica positiva y negativa, la ausencia de información preventiva, la crítica negativa y el feedback correctivo. Creemos que es importante que los entrenadores minimicen el uso de las críticas negativas y que no permitan la existencia de conversaciones inapropiadas entre los jugadores, ya que pueden favorecer consecuentemente acciones violentas de contacto entre ambos.

Comportamientos de los entrenadores de fútbol de clubs grandes

Los entrenadores de fútbol de clubs grandes favorecen un clima de entrenamiento positivo (fig. 3) con numerosas críticas positivas (n=322) y bromas dirigidas a los jugadores ( $\mathrm{n}=103$ ). También existe una creciente preocupación de salud por parte de estos entrenadores con ejercicios dirigidos en el trabajo de capacidades físicas con feedbacks correctivos ( $\mathrm{n}=98)$, así como consejos de prevención de lesión $(\mathrm{n}=35)$. Por otra parte son minoritarias las críticas negativas ( $\mathrm{n}=97$ ), la permisión de conversaciones inapropiadas $(\mathrm{n}=28)$ y las amenazas a los jugadores $(n=23)$.

En las cadenas de conductas que obtenemos de estos dendogramas (fig.4) constatamos la alternancia entre el feedback correctivo asociado a las informaciones sobre prevenciones, y la combinación entre las bromas a los jugadores con la crítica positiva de un clima agradable. Esto representa la preocupación por transmitir información sobre la forma correcta de realizar ejercicios destinados a la formación de capacidades físicas, así como para corregir malas actuaciones en su curso.

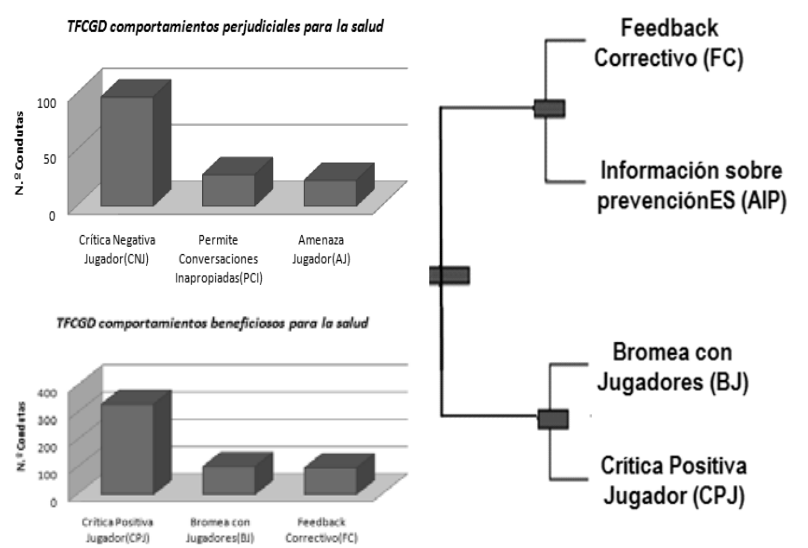

Figura 3. Conductas de los entrenadores de fútbol de Figura 4. Dendograma de los entrenadores de fútbol clubs grandes de clubs grandes

Comportamientos de los entrenadores de fútbol sala de clubs pequeños

En esto entrenadores (fig. 5) vemos que no existen diferencias entre el número de críticas negativas ( $\mathrm{n}=213$ ) y el de críticas positivas ( $\mathrm{n}=239)$. Asi constatamos que las críticas negativas promueve un climas enrarecido que incentiva la aparición de: conversaciones inapropiadas que son permitidas por el entrenador $(n=66)$, y amenazas consecutivas contra estos jugadores ( $\mathrm{n}=60$ ). También aparecen residualmente la preocupación y cuidado de jugadores más desprotegidos ( $\mathrm{n}=15$ ) y la emisión de feedback correctivo $(n=13)$ en algunos ejercicios en el entrenamiento físico.

La secuencia de las conductas (fig. 6) manifiesta la relación entre la ausencia de una información preventiva y la crítica negativa de un jugador concreto y del grupo que se combina con la ausencia de feedback correctivo y amenazas a los jugadores. También vemos algunas conductas de crítica positiva que va acompañada de actuaciones que permiten las conversaciones inapropiadas que, a su vez, se relacionan con críticas negativas.

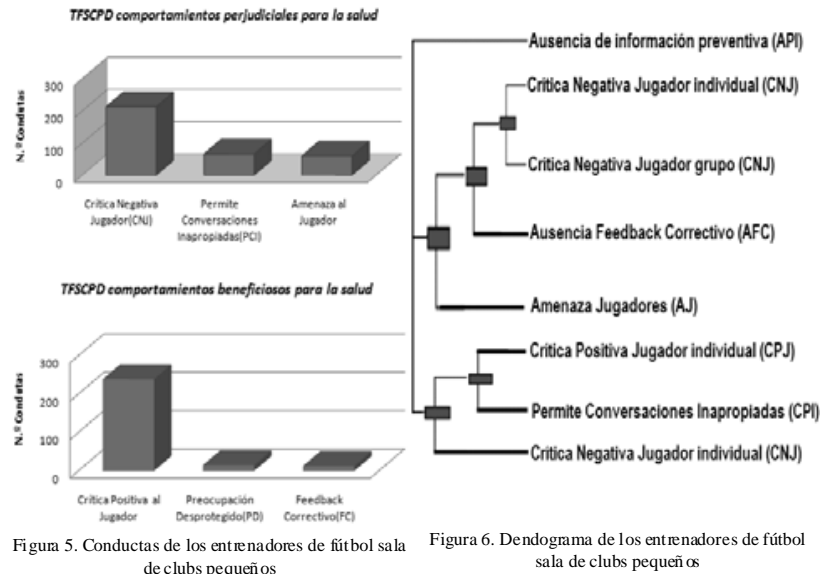

Comportamientos de los entrenadores de fútbol sala de clubs grandes

En estos entrenadores la crítica positiva $(n=214)$ es la sobresaliente, aunque no informan a sus jugadores sobre los riesgos y prevenciones de lesiones $(\mathrm{n}=28)$ en los ejercicios de capacidades físicas. De esta manera, surge la necesidad de emitir feedbacks $(n=43)$ en algunas ocasiones y en otras no $(\mathrm{n}=21)$. Esto revela una inconstancia en lo que se refiere a conductas que tienen como objetivo proteger la integridad física. Sobre la relación entre el entrenador de atletas, podemos ver que los entrenadores bromean $(n=29)$ en un estrechamiento de relaciones positivas entre el entrenador y jugador.

Un análisis más minucioso de estas conductas nos permite relacionarlas secuencialmente para ver (fig. 8) la relación entre las criticas positivas, dirigidas primero al grupo y posteriormente a un jugador en concreto, con la aparición de crítica negativa individuales que estas están relacionadas con más críticas positivas, está vez al revés, primero a un jugador individual y posteriormente a todo el grupo.

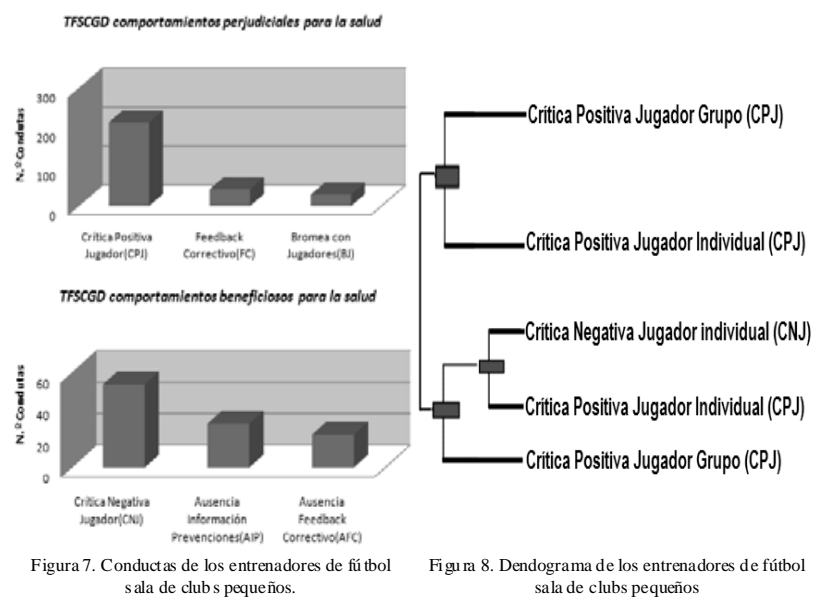

\section{Discusión y conclusiones}

Los entrenadores tienen una gran influencia y responsabilidad sobre los participantes de edades tempranas y pueden inculcar hábitos saludables fomentando el gusto por la práctica de actividad física (Rowland, 2007). Por ello es de gran importancia analizar y revisar la actuación de los entrenadores en su cometido educativo (Telama, Yang, Hirvensalo \& Raitakari, 2006). En nuestro estudio hemos comprobado que todos los entrenadores, sin distinción de modalidad -fútbol/fútbol sala- o el tamaño de la organización deportiva -pequeño/grande- manifiestan tanto conductas que son beneficiosas como perjudiciales para la salud de sus jugadores.

Si profundizamos en los resultados constatamos que son los entrenadores de los clubes grandes, tanto de fútbol como de fútbol sala, los que revelan mayores conductas beneficiosas -la crítica positiva(CPJ), la 
reprobación de contactos violentos (RCFV), y de actitudes de ayuda hacia los jugadores desprotegidos (PD)- en comparación con los entrenadores de estas dos modalidades de clubs pequeños.

Con referencia a las conductas nocivas para la salud de los jugadores -la crítica negativa a los jugadores (CNJ), la permisividad de conversaciones inapropiadas (RCI), las amenazas dirigidas a los jugadores (AJ), la falta de preocupación para con los desprotegidos (APD), y la ausencia de feedback correctivo (FC)- se revelan como resultados inversos al comprobar que son los entrenadores de fútbol de clubs pequeños aquellos que manifiestan más conductas perjudiciales para la salud de sus jugadores ( $\mathrm{n}=726)$, y que son los entrenadores de fútbol sala de clubs grandes los que las manifiestan en menor número ( $\mathrm{n}=142)$.

Podemos concluir que la dimensión, estructura y complejidad de la organización deportiva parece influir en la actuación de los entrenadores, así en los clubes grandes encontramos más cantidad de actitudes favorables hacia conductas previsoras de salud que en los clubs pequeños tanto en la modalidad de fútbol como de fútbol sala. Sin embargo, si queremos diferenciar y particularizar las estrategias más utilizadas encontramos la corrección positiva o feedback correctivo (FC), para corregir y orientar la realización de los ejercicios, como el comportamiento más beneficioso en pro de la salud de los practicantes y el uso de las críticas negativas (CNJ), dirigidas delante de actuaciones desfavorables de los jugadores, como el comportamiento nocivo más registrado.

También podemos afirmar que son los entrenadores de los clubs más grandes los que revelan una mayor variedad en la tipología de las conductas beneficiosas para la salud de sus jugadores, a diferencia de los entrenadores de fútbol sala de clubs de tamaño pequeño que son los que manifiestan menor variedad de conductas registrada. Esto viene a reforzar nuestras conclusiones anteriores y puede ser debido, tal como apuntan otros autores (Mesquita, Farias, Rosado, Pereira \& Moreno, 2008), a la diferencia en los niveles de preparación y formación de los técnicos con el consiguiente peligro para sus jugadores en cuanto a la posible aparición de lesiones, abandonos prematuros y dificultades futuras con relación a la salud. Por tanto sería necesario replanteary revisar la forma de estructurar las organizaciones y los programas deportivos dedicados a los noveles deportistas en proceso de formación (Weinberg \& Gould, 1995).

\section{Agradecimientos}

Este estudio forma parte de las investigaciones:

- Avances tecnológicos y metodológicos en la automatización de estudios observacionales en deporte que ha sido subvencionado por la Dirección General de Investigación, Ministerio de Ciencia e Innovación (PSI2008-01179), durante el trienio 2008-2011 y del grupo de investigación consolidado de la Generalitat (2009-2013). - Grup de recerca i innovació en dissenys (GRID). Tecnologia i aplicació multimèdia i digital als dissenys observacionals.

\section{Referencias}

Añó, V.(1997). Planificacióny Organización del entrenamiento juvenil. Madrid: Gymnos. Anguera, M. (1990). Metodología observacional. En: J. Arnau, M. Anguera y J. Gómez (Eds.), Metodología de la investigación en Ciencias del Comportamiento (pp. 125-236). Murcia: Secretariado de Publicaciones de la Universidad de Murcia.

Anguera, M. (2003). Metodología básica de observación en fútbol. In T. Ardá (Ed.), Metodología de la enseñanza del fútbol (pp. 303-324). Barcelona, España: Paidotribo.

Anguera, M., Blanco, A., \& Losada, J. (2001).Diseños observacionales, cuestión clave en el proceso de la metodología observacional. Metodologia de las ciencias del comportamiento, 3(2), 135-160.

Anguera, M., Blanco, A., Mendo, A., \& Losada, J. (2011). Diseños observacionales: ajuste y aplicación en psicología del deporte. Cuadernos de Psicologia del Deporte Dirección General de Deportes, 2, 63-76.

Ara, I., Vicente-Rodriguez, G, Perez-Gomez, J., Jimenez-Ramirez, J., Serrano-Sanchez, J.A Dorado. C., \& Calbet. JAL (2006). Influence of extracurricular sport activities on body composition and physical fitness in boys: a 3-year longitudinal study. International journal of obesity, 30, 1062-1071.

Bompa, T. (1999). Periodization. Theory and Methodology of training. Champaign IL: Human Kinetics.

Biddle, S., Sallis, J., \& Cavill, N. (1998). Young and active? Young people and healthenhancing physical activity: Evidence and implications. Health Education Authority Symposium. London.
Buceta, J. (2001). Comportamento do Treinador de Jovens no Treino. In: CEFD (Eds), Melhores treinadores para uma melhor prática. Seminário Internacional Treino de Jovens. Lisboa: Portugal.

Camerino, O., Chaverri, J., Anguera, M.T., \& Jonsson, G.K. (2012). Dynamics of the game in soccer: Detection of T-patterns. European Journal of Sport Science, 12(3), 216224.

Castellano, J., Mendo, A., Gómez, P., Fontetxa, E., \& Bueno, I. (2000). Sistema de codificación y análisis de la calidad del dato en el fútbol de rendimento. Psicothema, 12(4), 635-641.

Cavill N. (2001). Walking \& Health: making the links. World Transport Policy \& Practice, 7(4), 33-39.

Chelladurai, P. (1990). Leadership in sports: A review. International Journal of Sport Psychology, 21, 328-354

Cruz, J., \& Gomes, A. (1996). Liderança de equipas desportivas e comportamento do treinador. In J. F. Cruz (Ed.), Manual de Psicologia do Desporto (pp. 389-409). Braga: SHO - Sistemas Humanos e Organizacionais.

Devís, J., Beltrán, J.V., \& Fox· K (Published online 2013). Exploring socio-ecological factors influencing active and inactive Spanish students in years 12 and 13. Sport, Education and Society. Recuperado de internet en la dirección: http://dx.doi.org/ 10.1080/13573322.2012.754753

Fairclough, S.J., Boddy, L.M., Mackintosh, K. A., Valencia-Peris, A., \& Ramirez-Rico, E. (in press). Weekday and weekend sedentary time and physical activity in differentially active children, Journal of Science and Medicine in Sport

Ford, P., Yates, I., \& Williams, M. (2010). An analysis of practice activities and instructiona behaviours used by youth soccer coaches during practice: Exploring the link between science and application. Journal of Sports Sciences, 28(5), 483-495.

Gabín, B., Camerino, O., Anguera, M. T., \& Castañer, M. (2012). Lince: multiplatform sport analysis software. Procedia-Social and Behavioral Sciences, 46, 4692-4694.

Gioftsidou, A. y Malliou, P. (2006). Preventing lower limb injuries in soccer players. Strength and Conditioning Journal, 28(1), 10-13

Gorely, T., Biddle, S., Marshall, S., \& Cameron, N. (2009). The prevalence of leisure time sedentary behaviour and physical activity in adolescent boys: An ecological momentary assessment approach. International Journal of Pediatric Obesity, 4(4), 289-298.

Haskell, W., Lee, I, Pate, R, Powell, K, Blair, S., Franklin, B., Macera, C., Heath, G, Thompson, P., \& Bauman, A. (2007). Physical activity and public health: Update recommendation for adults from the American College of Sports Medicine and the American Heart Association. Medicine and Science in Sport and Exercise, 39(8), 1423-1434.

Kibler, W.B. (1995). Injuries in adolescent and preadolescent soccer players. Medicine and Science in Sports and Exercise, 25(12), 1330-1332

Janssen, I., \& LeBlanc, A. (2010). Systematic review of the health benefits of physica activity and fitness in school-aged children and youth. International Journal of Behavioral Nutrition and Physical Activity 7(40), 1-16.

Jonsson, GK., Anguera, M.T., Blanco-Villaseñor, A., Losada, J.L., Hernández-Mendo, A Ardá, T., Camerino, O., \& Castellano, J. (2006). Hidden patterns of play interaction in soccer using SOF-CODER. Behavior Research Methods, 38(3), 372- 381

Jonsson, G.K., Anguera, M.T., Sánchez-Algarra, P., Olivera, C., Campanico, J., Castañer, M., Dinušova, M., Chaverri, J., Camerino, O., \& Magnusson, M. S. (2010). Application of T-Pattern Detection and Analysis in Sports Research. The Open Sports Sciences Journal, 3, 95-104.

Magnusson, M.S. (1996). Hidden real-time patterns in intra- and inter-individual behavior European Journal of Psychological Assessment, 12(2), 112-123.

Magnusson, M.S. (2000). Discovering hidden time patterns in behavior: T-patterns and their detection. Behavior Research Methods, Instruments, \& Computers, 32(1), 93-110.

Magnusson, M. S. (2006). Structure and Communication in Interaction. En G Riva, M. T Anguera, B. K. Wiederhold \& F. Mantovani (Eds.), From Communication to Presence: Cognition, Emotions and Culture Towards the Ultimate Communicative Experience (pp. 127- 146). Amsterdam: IOS Press.

Mesquita, I., Farias, C., Rosado, A., Pereira, F., \& Moreno, M.P. (2008). La presentación de las tareas en función de la formación académica de los entrenadores de fútbol. Motricidad.European Journal of Human Movement, 20, 119-134.

Olmedilla, O., Andreu, M.D., Ortín, F.J., \& Blas, A. (2009). Lesiones y fútbol base: un análisis en dos clubes de la Región de Murcia, Retos, 16, 63-66.

Ortega, F.B., Ruiz, J.R., Castillo M.J., \& Sjöström, M. (2008). Physical fitness in childhood and adolescence: a powerful marker of health. International journal of obesity, 32, 1-11.

Peiró, C. Devís, J., Beltrán, J.V., \& Fox K (2008). Variability of Spanish adolescents' physical activity patterns by seasonality, day of the week and demographic factors. European Journal of Sport Science , 8(3), 163-171.

Rivas, C., Romero, A., Pérez-Llantada, M., Llave, A., Portau, M., Molina, I., González, J., \& Garcia-Mas, A. (2012). Bienestar psicológico, salud general, autonomía percibida y lesiones en futbolistas. Revista de Psicología del Deporte, 21(2), 365-371.

Rowland, T. (2007). Physical Activity, Fitness, and Children. In: C. Bouchard, S. Blair, \& W. Haskell (Eds) Physical Activity and Health, (259-270) $\left(2^{\text {nd }}\right.$ ed.). United States: Human Kinetics.

Telama, R., Yang, X., Hirvensalo, M., \& Raitakari, O. (2006). Participation in Organized Youth Sport as a Predictor of Adult Physical Activity: A 21-year longitudinal study. Pediatric Exercise Science, 18(1), 76-88.

Weinberg, R. S., \& Gould, D. (1995). Fundamentos de psicología del deporte y el ejercicio físico. Barcelona: Ariel.

Wickel, E., \& Eisenmann, J. (2007). Contribution of youth sport to total daily Physical Activity among 6- to 12- years old boys. Medicine and Science in Sports and Exercise, 39(9), 1493-1500. 